\title{
Privacy and Control in Mobile Cloud Systems
}

\author{
Tariq Alwada'n \\ Faculty of Information Technology \\ The World Islamic \\ Sciences and Education \\ University Amman, Jordan
}

\author{
Omar Al-Zitawi \\ King Hussein Faculty of Computing \\ Sciences \\ Princess Sumaya University for \\ Technology, Amman-Jordan \\ Mohammed Almasarweh \\ Faculty of Information Technology \\ The World Islamic \\ Sciences and Education \\ University Amman, Jordan
}

\author{
Sufian Khawaldeh \\ Faculty of Information Technology \\ The World Islamic \\ Sciences and Education University \\ Amman, Jordan
}

\begin{abstract}
Cloud computing is a huge amount of distributed and dynamic sharing resources that are provided on request to clients over the Internet instead of having a local server or personal devices to deal with applications. Usually, cloud servers don't work at full power; means there's some processing power will be unused. To make use of this wasted power, it's possible to use the mobility which is used to migrate application software, data or jobs among cloud servers. The problem with such technology is the privacy. In this paper we proposed a policy management framework to protect the privacy while applying the mobility method in the cloud environment.
\end{abstract}

\section{General Terms}

Distributed Systems, Computer Resources.

\section{Keywords}

Cloud Computing, Mobility, HCI, Policy Management, Privacy.

\section{INTRODUCTION}

\subsection{Definition}

Cloud computing is a huge amount of distributed and dynamic resources that are provided on request to clients over the Internet. Various resources can be provided from computing power, storage and services that are provided on request to clients over the internet [13]. In general, the cloud computing resources situates in massive data storage centers and is managed by a third group, who offers computing infrastructures which can be approached from anywhere by anyone with internet services [9]. A lot of authors, such as [6, $11,15,10,14]$, draw up the fact that cloud computing provides computing power, software, storage on demand distributed over the internet and managing large scale of data. Figure (1) shows the architecture model for the cloud computing. Cloud services let individuals and businesses to utilize software and hardware that are organized by third parties at remote sites. Such examples of cloud benefits include social networking sites, online file storage and webmail. Because of the economic benefits for providers and users, cloud computing becomes popular as it increases the revenue for cloud provider and lowers the costs for cloud users $[2,17,7]$. The cloud computing system gains access to information and computer resources from anyplace that has a network connection [3].

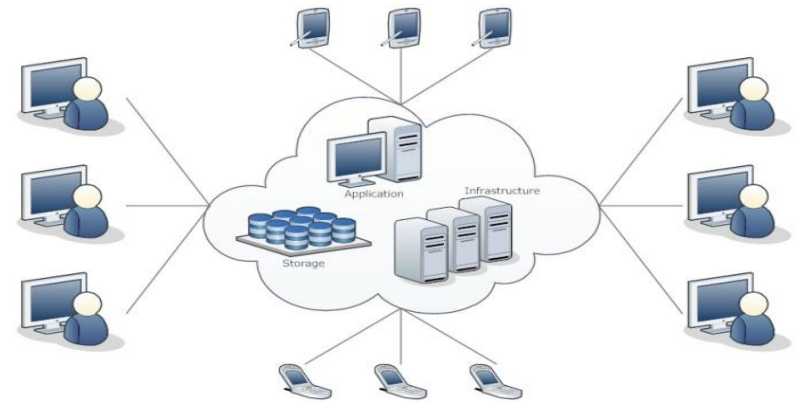

Fig. 1. Cloud Computing Environment [18]

There is a modern model theory that divides cloud computing into four recognized models. This model is called Cloud Computing Deployment Models [5, 12]. The model is called the Public model, where services and resources are accessible to the public by using the internet. This environment points up that a user has the ability to use only the required services and pay only for their use, but the system is organized and hosted by a third party. The main worry in this method of cloud environment is the security.

The second model is the Private one, in which services and resources are accessible within a private organization. This environment points up the advantages of integration, optimization of hardware deals and scalability. The main worry is the complexity, as this environment is organized and hosted by internal resources. Security is not a main issue compared to the public cloud as the services are reachable only through private and internal networks.

The Third one is the Community model, where services and resources of this method are shared by different organizations with a shared aim. It may be arranged by one of the organizations or a third party [16]. The last one is the Hybrid model. In this method mixes the methods from the private and public clouds, where resources can be utilized either in a public or a private cloud environment [19]. The advantages and the concerns are a combination of the earlier methods.

\subsection{Mobility}

Mobility is the capability to migrate, move or rearrange application software, data and/or jobs between cloud servers. This technology is called Computational Mobility. Mobility makes possible to complete the jobs and users requirements in cloud environment. Also, it assists cloud evolution, improves performance of operating applications by repositioning data to 
the intention host, consequently; reducing the communication consumption and solving the load balancing problems. Computational mobility can be called a control migration, data migration, link and object migration [4]. In this type of migration data and codes are allowed to migrate and execute on many systems among the network. Also it presents shifting execution control and the capability to link software elements at runtime as migrating from one system to another and back to the original system over again. There are two types of mobility. The first on is the Weak Mobility (WM), which allows code to migrate through the networks but without its execution states. The second type is the Strong Mobility (SM), in which the code and execution state are moved to start again at a new resource. Using this way can save running time, processor, registers and program counters.

The rest of the paper will be organized as follows. The next section describes the system architecture. This section includes the Cloud Computing Architecture and the Mobile Cloud Architecture. In section three, we discuss future possibilities and conclude the paper.

\section{SYSTEM ARCHITECTURE}

\subsection{Cloud Computing Architecture}

Figure (2) shows the components of the cloud computing system. Usually it is divided into two parts; the front end and the back end parts. The two parts are connected to each other through a network, normally the internet. The first side (front end side) is the side where the computer users, or clients, access the cloud computing system using computer (or computer network) and the applications required for this connection. Not all cloud computing systems own the similar user interface. The other side (the back end side) consists of various servers, computers and data storage systems that build the "cloud" of computing services environments. The organizer of the cloud system is a central server which is responsible for administrative, monitoring traffic and client requests to guarantee that the whole thing runs efficiently [20].

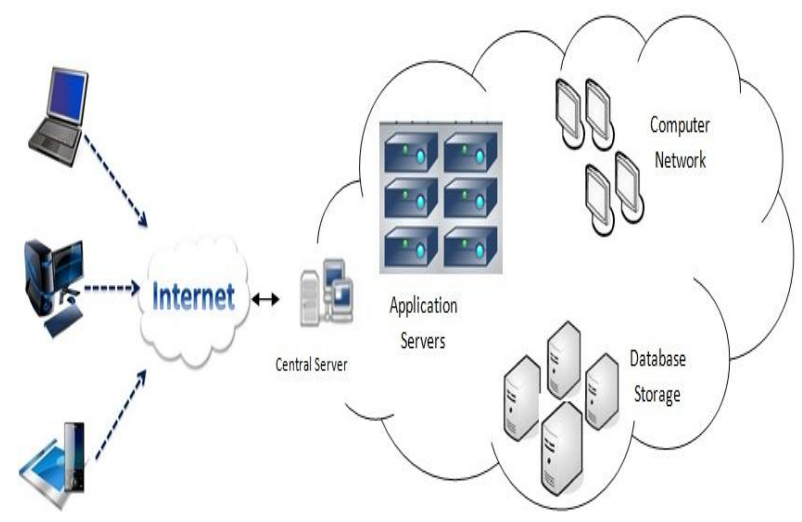

Fig. 2. Cloud Computing Architecture

\subsection{Mobile Cloud Architecture}

The central server in the cloud system follows a set of rules called protocols and utilizes particular software called middleware [20]. Middleware permits networked computers to speak to each other. Usually, servers don't operate at full capacity; means there's some processing power will be wasted. To make use of this wasted power, it's possible to divide the server into multiple virtual servers, each one running with its own operating system. This method is called server virtualization. The advantages of such method are maximizing the output of single servers and cut down the need for additional physical machines. Alwada'n in [1] introduced a mobile cloud framework that can support the mobility in the cloud system. In this framework, the central server has the ability to relocate jobs or data or even applications from one server to another depending on the new jobs requirements. To explain this process, let's assume that a new job comes to the cloud system and this job has specific requirements (application, data or hardware requirements) and these requirements are available only on some servers. In this case the central server can move, migrate, one or more running jobs with its execution code (strong mobility) to other available servers that can fulfill their requirements. These process of moving have created a chance for the new job to be process in the vacated server(s).

So, the mobility is done when a new job comes to the cloud system, the central server checks if this job can be processed in one of the available servers. When the central server reached to the result that no available server has the needed requirements to fulfill the new job, it asks the mobility server (one of the sub servers of the central server) to determine the busy servers that can process the new job and the availability to relocate any of the running jobs from those servers. Figure (3) shows this architecture. If the mobility server managed to relocate one of the running jobs, in this case the new job can be processed in the vacated server.

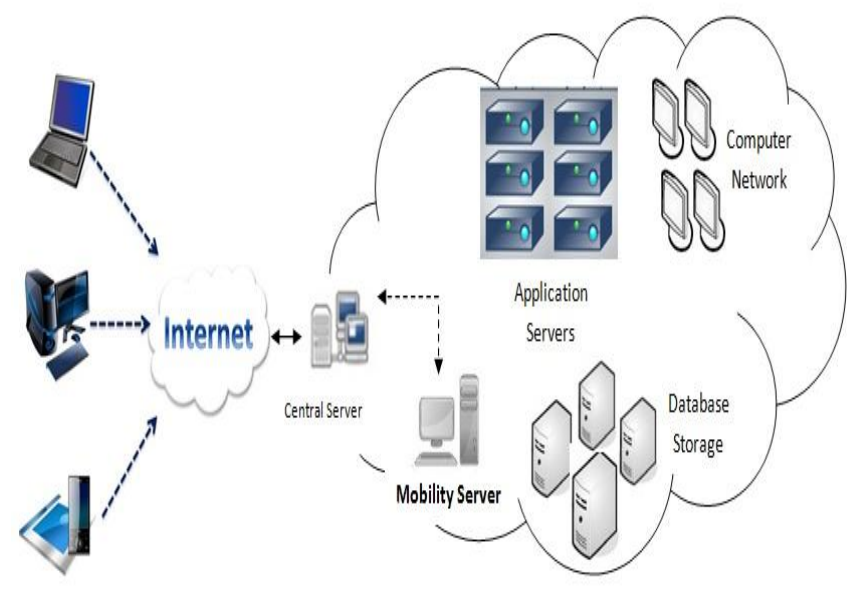

Fig. 3. Mobile Cloud Architecture

Figure (4) shows an example of the previous mobility process. In this figure Job (A) asked for a job from the cloud system, but only Server (1) can satisfy this job. The best way to solve this issue is to vacate this server from any running jobs inside and move Job (A) to it.

The infrastructure of the cloud normally depends on web models (over SSL) to establish and access account information for the external users, and allows them to change or reset their keys (passwords) with a new ones by email in what can be considered as unencrypted communication [13]. This means the privacy of the users' data and ability to give the permissions to handle this data to a specific domains or users by the original data owner is less than expected. 


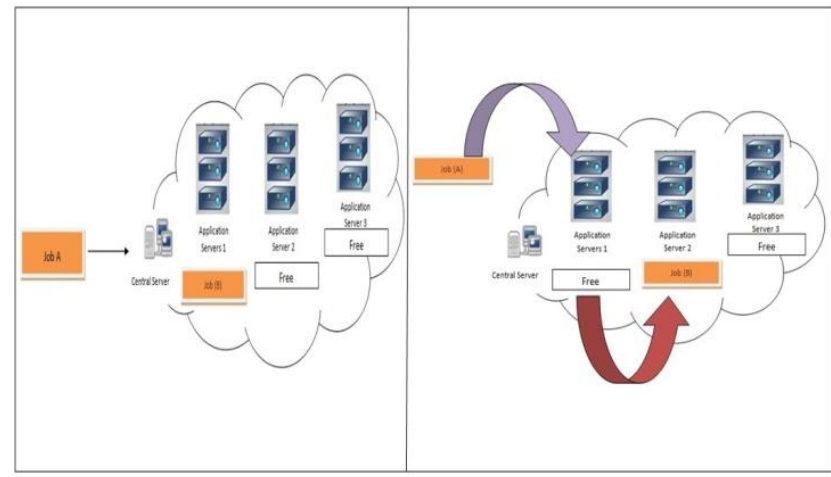

Fig.4. Cloud Mobility Example

Security is an essential element in cloud computing. For any Cloud environment, there should be methods to offer security, including authentication, authorisation and data encryption. It can be seen that the stages of anonymity and privacy provided by cloud to the external users will be less than the user of desktop in numerous situations [8]. Alwada'n and others in [21] proposed a framework that has a solution that may solve the privacy issue in the cloud system. This method is applied by giving the users the control over their data by sending their policies along with their job. Policies are groups of regulations, standards and practices written by one or more owners of jobs or administrators of resources about how their resources, jobs or data can be handled and used. Policies decide how a job should be done, how security is applied in a domain and how an organization organizes, secures and distributes their resources. That means the user policy should be taken into account when taking the final decision. Figure (5) shows how this framework works.

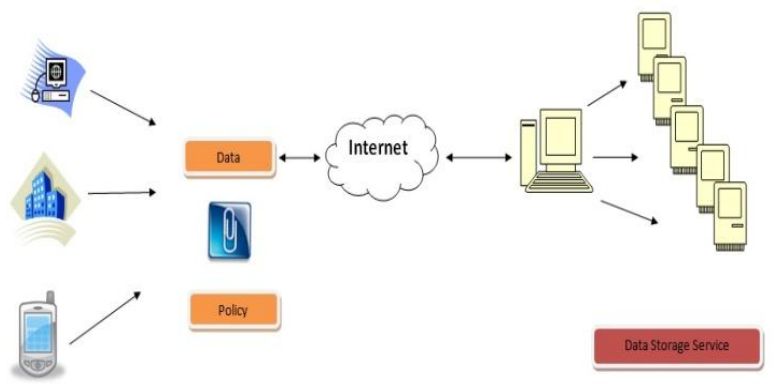

Fig. 5. Policy Framework [21]

We proposed a policy management framework to protect the privacy while applying the mobility method in the cloud environment. Our framework simply depends on sending the user's policy along with the user data and/or application(s). Before the owners send their data or applications, they choose the user and/or the organizations that allow their data to be sent to them. Also the owners can choose to which country their data allowed to be stored or manipulated. This policy that been sent along with the owners' data or application is traveled along with the data or application all the time. That means this policy should be applied all the time especially when applying the mobility model. Also, when there is a need to vacate one of the running jobs from one of the servers, the policy of the running job is checked by the mobility server to see if it's ok for the running job to be moved to the target server. If the movement agreed then the mobility server attaches the policy of the running job while sending that job to its new server. The same process is applied for data movements. Figure (6) shows this framework.
As shown in the Figure (6) when the users send their data or jobs to the cloud system, they send their policies as well. Also when Job (B) was vacated from Server (1) the mobility server sent the Job (B)'s policy along with the job to the new server (Server 2). Although mobility model has increased the traffic within the cloud system and the time needed to find the suitable server to process the cloud jobs, but it has given the cloud users and the cloud system the ability to control their data and job movements in the cloud system.

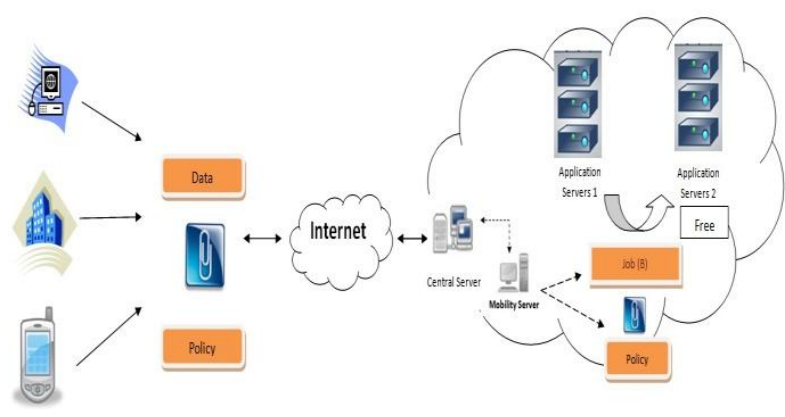

Fig. 6. Mobile Policy Framework

\section{CONCLUSIONS AND FUTURE WORK}

As cloud computing becomes one of the most modern technologies used at the moment, the need to use and take advantage of all of it resources without wasting its power is a serious problem for such technology. The mobility model has solved such a problem by migrating or relocating resources among cloud servers. The problem of such technology is the privacy. We presented in this paper a policy management framework to protect the privacy while applying the mobility method in the cloud environment.

\section{REFERENCES}

[1] Alwada'n, T.: Mobile cloud systems. CIT 19(1) (2014)

[2] Armbrust, M., Fox, A., Griffith, R., Joseph, A.D., Katz, R., Konwinski, A., Lee, G., Patterson, D., Rabkin, A., Stoica, I., Zaharia, M.: A view of cloud computing. Commun. ACM 53(4), 50-58 (Apr 2010), http://doi.acm.org/10.1145/1721654.1721672

[3] The Office of the Privacy Commissioner of Canada, O.: Introduction to cloud computing (October 2011), http://www.priv.gc.ca/resource/fs-

fi/02_05_d_51_cc_e.asp

[4] Cardelli, L.: Secure internet programming. chap. Abstractions for mobile computations, pp. 51-94. Springer-Verlag, London, UK (1999), http://portal.acm.org/citation.cfm?id=380171.380179

[5] De Chaves, S.A., Uriarte, R.B., Westphall, C.B.: Toward an architecture for monitoring private clouds. IEEE Communications Magazine 49(12), 130-137 (2011), http://dblp.uni-

trier.de/db/journals/cm/cm49.html\#ChavesUW11

[6] De Chaves, S.A., Westphall, C.B., Lamin, F.R.: Sla perspective in security management for cloud computing. In: Proceedings of the 2010 Sixth International Conference on Networking and Services. pp. 212-217. ICNS '10, IEEE Computer Society, Washington, DC, USA (2010), http://dx.doi.org/10.1109/ICNS.2010.36

[7] Chen, Y., Paxson, V., Katz, R.H.: Whats new about 
cloud computing security?Tech. Rep. UCB/EECS-20105, EECS Department, University of California, Berkeley (Jan 2010), http://www.eecs.berkeley.edu/Pubs/TechRpts/2010/EEC S-2010-5.html

[8] DELANEY, K.J., VARA, V.: Google plans service to store users' data (NOVEMBER 27, 2007), http://online.wsj.com/article/SB119612660573504716.ht $\mathrm{ml}$

[9] Foster, I., Zhao, Y., Raicu, I., Lu, S.: Cloud computing and grid computing 360-degree compared. 2008 Grid Computing Environments Workshop abs/0901.0(5), 1-10 (2008), http://arxiv.org/abs/0901.0131

[10] Foster, I., Zhao, Y., Raicu, I., Lu, S.: Cloud computing and grid computing 360-degree compared. 2008 Grid Computing Environments Workshop abs/0901.0(5), 1-10 (2008), http://arxiv.org/abs/0901.0131

[11] Heyman, T., Scandariato, R., Huygens, C., Joosen, W.: Using security patterns to combine security metrics. In: Proceedings of the 2008 Third International Conference on Availability, Reliability and Security. pp. 1156-1163. IEEE Computer Society, Washington, DC, USA (2008), http://dl.acm.org/citation.cfm?id=1371602.1371977

[12] Huth, A., Cebula, J.: The basics of cloud computing (2011), carnegie Mellon University. Produced for USCERT, a government organiztion

[13] Jaeger, P., Lin, J., Grimes, J.: Cloud computing and information policy: Computing in a policy cloud? Journal of Information Technology Politics 5(3), 269283

(2008), http://www.informaworld.com/openurl?genre=article \&d oi $=10.1080 / 19331680802425479 \&$ magic $=$ crossref
[14] Leavitt, N.: Is cloud computing really ready for prime time? Computer 42, 15-20 (January 2009), http://dx.doi.org/10.1109/MC.2009.20

[15] Mei, L., Chan, W.K., Tse, T.H.: A tale of clouds: Paradigm comparisons and some thoughts on research issues. In: Proceedings of the 2008 IEEE Asia-Pacific Services Computing Conference. pp. 464-469. IEEE Computer Society, Washington, DC, USA (2008), http: //dl.acm.org/citation.cfm?id=1487739.1487898

[16] Mell, P., Grance, T.: The nist definition of cloud computing ( draft ) recommendations of the national institute of standards and technology. Nist Special Publication 145(6), 7 (2011) http://csrc.nist.gov/publications/drafts/800-145/Draft-SP800-145_cloud-definition.pdf

[17] Services, A.W.: Aws economics center (May 2012), http://aws.amazon.com/economics/

[18] Singh, R.: Cloud computing from the home (December 2012), http://www.tutorialspoint.com/shorttutorials/cloudcomputing-from-the-home

[19] Sotomayor, B., Montero, R.S., Llorente, I.M., Foster, I.: Virtual infrastructure management in private and hybrid clouds. IEEE Internet Computing 13, 14-22 (September 2009),

//dl.acm.org/citation.cfm?id=1638588.1638692

http:

[20] Strickland, J.: How cloud computing works (April 2008), http://computerhowstuffworks.com/cloudcomputing/cloud-computing4.htm

[21] Tariq Alwada'n, Adel Hamdan Mohammad, H.A.: Privacy in cloud systems. CIT 19(1) (2014). 